\title{
Abnormal neural responses to harmonic syntactic structures in congenital amusia
}

Article

Accepted Version

Zhou, L., Liu, F., Jiang, J., Jiang, H. and Jiang, C. (2019)

Abnormal neural responses to harmonic syntactic structures in congenital amusia. Psychophysiology, 56 (9). e13394. ISSN 1469-8986 doi: https://doi.org/10.1111/psyp.13394 Available at https://centaur.reading.ac.uk/83397/

It is advisable to refer to the publisher's version if you intend to cite from the work. See Guidance on citing.

To link to this article DOI: http://dx.doi.org/10.1111/psyp.13394

Publisher: Wiley

All outputs in CentAUR are protected by Intellectual Property Rights law, including copyright law. Copyright and IPR is retained by the creators or other copyright holders. Terms and conditions for use of this material are defined in the End User Agreement.

\section{www.reading.ac.uk/centaur}

\section{CentAUR}

Central Archive at the University of Reading

Reading's research outputs online 


\title{
Abnormal neural responses to harmonic syntactic structures in congenital amusia
}

\author{
Linshu Zhou ${ }^{\mathrm{a}}$, Fang Liu ${ }^{\mathrm{b}}$, Jun Jiang ${ }^{\mathrm{a}}$, Hanyuan Jiang ${ }^{\mathrm{c}}$, Cunmei Jiang ${ }^{\mathrm{a}^{*}}$ \\ ${ }^{a}$ Music College, Shanghai Normal University, Shanghai, China \\ ${ }^{b}$ School of Psychology and Clinical Language Sciences, University of Reading, Reading, \\ $U K$ \\ ${ }^{c}$ Faculty of Humanities and Arts, Macau University of Science and Technology, Macau, \\ China
}

Running head: Processing harmonic musical structures in amusia

${ }^{*}$ Corresponding author at: Music College, Shanghai Normal University, 100 Guilin Road, Xuhui District, Shanghai 200234, China.

E-mail address: cunmeijiang@126.com (C. Jiang). 


\section{Abstract}

Harmonic syntactic structures are organized hierarchically through local and long-distance dependencies. The present study investigated whether the processing of harmonic syntactic structures is impaired in congenital amusia, a neurodevelopmental disorder of pitch perception. Harmonic sequences containing two phrases were used as stimuli, in which the first phrase ended with a half cadence and the second with an authentic cadence. In Experiment 1, we manipulated the ending chord in the authentic cadence to be either syntactically regular or irregular. Sixteen amusics and 16 controls judged the expectedness of these chords while their EEG waveforms were recorded. In comparison to the regular endings, irregular endings elicited an ERAN, an N5 and a late positive component in controls but not in amusics, indicating that amusics were impaired in perceiving harmonic syntactic structures induced by local dependencies. In Experiment 2, we manipulated the half cadence of the harmonic sequences to examine the processing of harmonic syntactic structures induced by long-distance dependencies. An ERAN-like response and an N5 were elicited in controls but not in amusics, suggesting that amusics were impaired in processing long-distance syntactic dependencies. Furthermore, for controls, the neural processing of local and long-distance syntactic dependencies was correlated in the late (as indexed by the N5) but not in the early stage. These findings indicate that amusics are impaired in the detection of syntactic violations and subsequent harmonic integration. The implications of these findings in terms of hierarchical music-syntactic processing are discussed. 
Keywords: Congenital amusia, Harmonic syntax, Hierarchical structure, Local dependency, Long-distance dependency 


\section{Introduction}

Music and language are unique to human beings. They both contain structural organizations based on syntactic principles (Koelsch et al., 2013; Patel, 2008). For example, in the sentence "the boy who ran the fastest won the prize", the subject relative clause "who ran the fastest" is nested in the main clause "the boy won the prize", which creates the long-distance dependency between syntactic constituents ("the boy" and "won"). Such syntactic structure constitutes a hierarchy, which refers to an organization of items based on their importance (Krumhansl, 1990). Hierarchy is considered to be a distinctive property of syntax in language (Fitch \& Hauser, 2004; Friederici et al., 2006; Hauser, Chomsky, \& Fitch, 2002).

Similar to language, tonal music is "syntactic" in the sense that perceptually discrete elements (such as tones or chords) are combined into musical sequences based on hierarchical relationships (Bigand et al., 2014). Typically, musical hierarchy can be represented by the perceived stabilities of musical events (Krumhansl, 1990). The more important they are, the more stability listeners feel (Bigand, 1993; Krumhansl, 1990). Specifically, tonic chords are perceived as the most stable, followed by dominant and subdominant chords, and then the remaining diatonic chords. Non-diatonic chords are perceived as the most unstable (Bharucha \& Krumhansl, 1983). Taking the musical period as an example, a period usually contains two phrases, where the first phrase ends with a dominant chord (V) while the second phrase ends with a tonic chord (I). As shown in Figure 
1, hierarchical syntactic structures are represented in both long-distance and local dependencies. For the long-distance dependency, as with language, the dominant chord at the end of the first phrase needs to be completely resolved by the tonic at the end of the next phrase, owing to the fact that the tonic is perceived as more stable than the dominant chord. For the local dependency, at the ending of the period, the progression of dominant and tonic chords would be perceived more stable than that of dominant and supertonic chords. This is because the tonic is perceived as more stable than the dominant chord, whereas the dominant chord is perceived as more stable than the supertonic chord (Krunmhansl, 1990), the latter of which needs to be recursively expanded to create a progression of dominant and tonic chords in the end (Lerdahl \& Jackendoff, 1987; Rohrmeier, 2011). According to music theory, ending on the dominant chord for the first phrase could be named as a half cadence, while the succession of the dominant and tonic chords at the end of a period is named as an authentic cadence (or full cadence). If we defined the succession of the dominant and tonic chords in the authentic cadence as a local hierarchical relation, then the dominant in the half cadence and the tonic in the authentic cadence would constitute a long-distance dependency of hierarchical syntactic structures.

Insert Figure 1, about here.

Previous studies have investigated the neural processing of music-syntactic structures induced by local and long-distance dependencies, respectively. In particular, electroencephalography (EEG) studies have shown that syntactic violations of local 
dependency in the authentic cadence elicited an early right anterior negativity (ERAN) and a subsequent late negativity (the so-called N5) (e.g., Kolesch et al., 2000; Koelsch \& Jentschke, 2010). While the ERAN reflects the detection of syntactic violation at the early stage (e.g., Kolesch et al., 2000; Koelsch \& Jentschke, 2010), the N5 reflects the processes of harmonic integration (Koelsch et al., 2000; Loui et al., 2005) or the processing of meaning information (Koelsch, 2011, 2012; Steinbeis \& Koelsch, 2008) in the later stage. These effects are specific to musical syntactic processing. Taking the ERAN as an example, apart from the involvement in processing harmonic syntactic structures, it is also involved in the detection of syntactic violation in rhythmic structures (L. Sun et al., 2018), which is different from the mismatch negativity (MMN, Alain, Achim, \& Woods, 1999; Tervaniemi et al., 2001) elicited by non-syntactic violations of expectations (such as pitch deviants in the oddball paradigm) (Kolesch, 2009).

Unlike the investigations of the syntactic processing induced by local dependencies, only one EEG study refers to the syntactic processing induced by long-distance dependencies (Koelsch et al., 2013). It has been demonstrated that irregular long-distance dependencies evoked an ERAN-like response and an N5 in both musicians and nonmusicians, which reveals that even musically untrained listeners could perceive long-distance dependencies in musical structures (Koelsch et al., 2013). Overall, these findings suggest that similar neural activities are associated with the processing of local and long-distance dependencies in musical syntactic structures. However, no study has yet directly compared between the processing of local and long-distance dependencies in hierarchical music-syntactic structures. 
Given the important role pitch plays in harmonic syntax, it is crucial to understand whether low-level pitch processes (such as pitch discrimination) are directly linked to higher-level pitch processes (such as harmonic syntactic processing). Congenital amusia (hereafter amusia) is a neurodevelopmental disorder of pitch perception, which is not attributed to brain lesions, hearing loss, lack of exposure to music, or any cognitive or socioaffective disturbance (Ayotte, Peretz, \& Hyde, 2002; Peretz et al., 2002). It remains unclear whether pitch processing deficits in amusia extend to the processing of harmonic syntactic structures. Examining the neural processing of harmonic syntactic structures in amusia would provide insight into whether there exists a neural network specific to musical pitch processing (Norman-Haignere et al., 2016; Zhou et al., 2017).

Previous studies have demonstrated that amusics are impaired in explicit pitch discrimination and identification (e.g., Foxton et al., 2004; Hyde \& Peretz, 2004; Jiang et al., 2010, 2011; Liu et al., 2010), despite showing intact implicit pitch processing (e.g., Omigie et al., 2012). The dissociation between implicit and explicit performance has also been reported in musical structural processing in both behavioral and EEG studies. Behaviorally, amusics failed to perceive harmonic syntactic structures based on the local dependency in the authentic cadence, being unable to distinguish a tonic chord from a Neapolitan chord (Jiang, Liu, \& Thompson, 2016), despite showing intact implicit processing of such structures (Tillmann, Gosselin, Bigand, \& Peretz, 2012). Using event-related potentials (ERPs), Zendal et al. (2015) found that amusics elicited an ERAN in response to out-of-key and out-of-tune notes in melodies in the implicit but not in the explicit task, although a recent study by Y. Sun 
et al. (2018) showed that amusics failed to elicit an ERAN for out-of-key notes in melodies even in an implicit task. Although the detection of out-of-key and out-of-tune notes in the studies by Zendal et al. (2015) and Y. Sun et al. (2018) required sensitivity to tonal hierarchy, these notes might also represent auditory sensory violations in melodies (Bigand et al., 2003; Koelsch et al., 2007). In order to rule out the possible confounding effect of auditory sensory violations associated with out-of-key and out-of-tune notes, it is necessary to design a more carefully controlled study to further examine the neural processing of syntactic structures in amusia, e.g., by using in-key notes or chords.

The present study thus examined the impact of amusia on the neural processing of harmonic syntactic structures induced by both local and long-distance dependencies. First, given the dissociation between implicit and explicit performance reported in previous studies (e.g., Omigie et al., 2012; Tillmann et al., 2016a; Zendal et al., 2015), we employed explicit rather than implicit tasks in order to pinpoint the neural origin of the anomaly in amusia. Second, the present study contained two experiments. Two phrases were used in both experiments, where the first phrase ended with a half cadence and the second phrase ended with an authentic cadence. We manipulated the syntactic regularity of the local dependency in the authentic cadence (Experiment 1) and the syntactic regularity of the long-distance dependency between the dominant in the half cadence and the final tonic (Experiment 2). This design enabled us to compare the patterns of ERPs elicited by local and long-distance syntactic dependencies from the two experiments. We predicted that the amusic brain would show atypical responses to harmonic syntactic structures, based on the evidence that amusics 
are impaired in explicitly perceiving harmonic syntactic structures at the behavioral level (Jiang et al., 2016).

\section{Experiment 1}

In Experiment 1, we investigated the impact of amusia on the processing of hierarchical syntactic structures induced by local dependency. We manipulated the ending chord (tonic versus supertonic chords) of harmonic sequences, creating syntactic-regular versus -irregular conditions. Unlike the tonic ending, the supertonic ending cannot hierarchically prolong the first chord of the sequence or close the established dominant that remained open at the end of the half cadence (Lerdahl \& Jackendoff, 1983; Rohrmeier, 2011) (Figure 2 and Audio Files S1 and S2). Moreover, the use of the supertonic prevented us from introducing any out-of-key note or new chord function (Jentschke, Friederici, \& Koelsch, 2014; Jentschke \& Koelsch, 2009; Koelsch, Jentschke, Sammler, \& Mietchen, 2007). In order to rule out the ERP effects elicited by sensory novelty, we also controlled for the frequency of occurrence of the notes in the regular and irregular chords. Given that the ERAN and N5 are supposed to reflect the detection of syntactic violation and subsequent harmonic integration (e.g., Koelsch et al., 2000; Koelsch \& Jentschke, 2010), we predicted that the irregular syntactic structures that are induced by the ending chord of the supertonic would elicit an ERAN and an N5 in controls but not in amusics.

Insert Figure 2, about here. 


\subsection{Method}

\subsubsection{Participants}

Table 1 summarises the demographics of the participants. Sixteen amusics and 16 matched controls were recruited through advertisements in the bulletin board systems of universities in Shanghai, China. Participants' musical abilities were measured by the Montreal Battery of Evaluation of Amusia (MBEA; Peretz, Champod, \& Hyde, 2003), which consists of six subtests assessing perception of scale, interval, contour, rhythm, and meter in music, as well as recognition memory of musical melodies. Before being invited to the lab for testing, all potential amusics self-reported that they had difficulty in carrying a tune when singing and in differentiating melodies during musical listening. The potential controls self-reported that they were able to sing in tune and differentiate melodies during musical listening. Participants were diagnosed as amusic if they scored $65 \%$ or below on the composite score of the three pitch-based subtests, i.e., scale, contour, and interval subtests (Liu et al., 2010), and below 78\% correct on the MBEA global score, which represents two standard deviations below the mean score of controls (Liu et al., 2010; Peretz et al., 2003). We also calculated the d' of the MBEA for each participant, and the d' for each amusic was below the cutoff of 1.23 reported by previous studies (Henry \& McAuley, 2013; Pfeifer \& Hamann, 2015). 
As shown in Table 1, the two groups were matched on age and years of education but differed significantly in scores of the MBEA and pitch discrimination thresholds. All participants were native speakers of Mandarin, right-handed, with normal hearing, and no history of psychiatric or neurological diseases. Although students in China usually attend a 45-min music lesson per week as part of the school curriculum, none of our participants had received extracurricular training in music. Hand dominance was assessed by the Edinburgh Handedness Inventory (Oldfield, 1971). Ethical approval was obtained from Shanghai Normal University, and all participants signed a written consent form before the experiments were conducted.

Insert Table 1, about here.

\subsubsection{Stimuli}

Two original chord sequences were transposed to 12 major keys, resulting in 24 different sequences. Each harmonic sequence contained two phrases. The first phrase ended with a half cadence while the second phrase ended with an authentic cadence. Each of the 24 sequences consisted of two different versions. The syntactic-regular version ended on a tonic chord, whereas the syntactic-irregular version ended on a supertonic chord (see Figure 2). The stimuli thus consisted of 48 chord sequences, of which 24 were syntactically regular and 24 syntactically irregular. 
Each of the 48 sequences was presented 4 times, resulting in 192 sequences for the entire experiment. Presentation time of the chords was $500 \mathrm{msec}$, except for the fifth and the final chords that lasted $1000 \mathrm{msec}$. Sequences were created using Cubase 5.1 (Steinberg Media Technologies GmbH, Hamburg, Germany), exported with the grand piano timbre (Galaxy Vienna Grand Library), with $44.1 \mathrm{kHz}$ sampling rate and 16-bit resolution. The loudness was normalized with Adobe Audition CS 6 at an approximate intensity of $68 \mathrm{~dB}$ SPL.

\subsubsection{Procedure}

The sequences were pseudorandomized and presented over two blocks of 96 trials through two loudspeakers positioned to the left and right of the participant. After the presentation of each chord sequence, participants were instructed to decide whether the progression of the chords was consistent with their expectation by pressing one of the two response buttons. Consistent with previous ERP studies (e.g., Koelsch et al., 2007; Kolk et al., 2003), the association between hand side (left or right) and the response (yes or no) was counterbalanced across participants in each group. The first block began with a practice session of 6 trials to familiarize the participants with the procedure.

\subsubsection{Electroencephalography (EEG) recording and analysis}

EEG data were recorded from 64 standard scalp locations (International 10-20 system), digitized at a rate of $500 \mathrm{~Hz}$, with a $0.05 \mathrm{~Hz}$ low cutoff filter and a $100 \mathrm{~Hz}$ high cutoff filter. The data were referenced off-line to the algebraical mean of left and right mastoid electrodes, 
filtered offline with a band-pass filter of 0.1 to $30 \mathrm{~Hz}$ (24-dB/oct slope). Trials were averaged offline with an epoch length of $1200 \mathrm{msec}$, including a baseline from $200 \mathrm{msec}$ to $0 \mathrm{msec}$ before the target onset. Trials with artifacts exceeding the amplitude of $\pm 75 \mu \mathrm{V}$ in any channel were rejected (Vaughan-Evans et al., 2014). On average, 12.6\% of all trials were rejected, and 84 trials $(S D=7)$ were retained per condition, with rejections being similarly distributed across groups and conditions $(F \mathbf{S}<1)$. After the time window was selected, the amplitudes were averaged under each condition for each participant.

Consistent with previous music-syntactic studies (e.g., Kolesch et al., 2007;

Poulin-Charronnat, Bigand, \& Koelsch, 2006), electrodes were grouped into four separate regions of interest (ROIs): left anterior (AF3, F1, F3, F5, FC1, FC3, FC5), right anterior (AF4, F2, F4, F6, FC2, FC4, FC6), left posterior (PO3, P1, P3, P5, PO1, PO3, PO5), and right posterior (PO4, P2, P4, P6, PO2, PO4, PO6). To test for specific patterns of scalp distribution, anterior and posterior ROIs established the factor anteriority, and left and right ROIs established the factor hemisphere.

Repeated measures ANOVAs were conducted. Group (amusics, controls) was considered as a between-subjects factor, whereas regularity (regular, irregular), anteriority (anterior, posterior), and hemisphere (left, right) were considered as the within-subjects factors. The mean amplitude of the respective electrodes in each region of interest was computed for analysis. Only the significant effects containing the main experimental variables (regularity and group) were reported. Overall ANOVAs were followed up by 
simple effects tests if there were significant two-way interactions between the main experimental variables.

\subsection{Results and discussion}

\subsubsection{Behavioral results}

Figure 3 shows the percentages of correct judgments $( \pm 1 S E M)$ for the regular and irregular sequences in controls and amusics, respectively. A repeated measures ANOVA with regularity as the within-subjects factor and group as the between-subjects factor revealed a main effect of group, $F_{(1,30)}=19.21, p<.001$, partial $\eta^{2}=.39$, as controls $(M=75.75 \%, S D=$ 16.78) achieved higher accuracy than amusics $(M=53.81 \%, S D=11.17)$. The overall performance of amusics did not differ significantly from the chance level $\left(t_{(15)}=1.37, p=.19\right.$; one-sample $t$-test). Further analysis showed that performance of amusics was above the chance level in the regular condition $\left(t_{(15)}=2.83, p=.01\right.$, Cohen' $d=0.71$; one-sample $t$-test $)$ but not in the irregular condition $\left(t_{(15)}=-0.79, p=.44\right.$; one-sample $t$-test $)$. The main effect of regularity $\left(F_{(1,30)}=3.00, p=.09\right)$ and the interaction between group and regularity $\left(F_{(1,30)}=\right.$ 2.02, $p=.17$ ) were not significant.

Insert Figure 3, about here.

Bivariate correlation analysis showed that the accuracy was not significantly correlated with the global MBEA scores, pitch detection thresholds, or pitch direction discrimination 
thresholds for either amusics or controls (all $p \mathrm{~s}>.14$ ). Furthermore, no significant correlation was found when accuracy rates in the regular and irregular conditions were considered separately, for either amusics or controls (all $p \mathrm{~s}>.07$, see Table S1, Supplemental materials for details).

\subsubsection{Electrophysiological results}

Figure 4 shows the electric brain responses to harmonically regular and irregular chord sequences at four representative electrodes. For controls, irregular chords elicited negative potentials with an approximate onset of $110 \mathrm{msec}$ and an approximate peak latency of 230 msec. However, the early negative potentials were not observed in amusics. Furthermore, irregular endings also elicited fronto-central negative potentials and posterior positive potentials at a late time window $(450-700 \mathrm{msec})$ in controls but not in amusics. Based on visual inspection and previous research on the ERAN and N5 (Koelsch et al., 2000; Koelsch et al., 2007), a time window from 110 to $230 \mathrm{msec}$ after the onset of final chords was selected for the analysis of the ERAN, and a time window from 450 to $700 \mathrm{msec}$ was selected for the analysis of the N5.

Insert Figure 4, about here.

An ANOVA with factors of group, regularity, anteriority, and hemisphere for the early time window $(110-230 \mathrm{msec})$ revealed an effect of regularity, $F_{(1,30)}=6.08, p=.02$, partial $\eta^{2}=.17$, as irregular endings elicited an ERAN as compared to regular endings. There was 
also an interaction between group, regularity, and hemisphere, $F_{(1,30)}=6.13, p=.02$, partial $\eta^{2}=.17$, owing to the fact that the ERAN with a right-hemispheric weighting was elicited in controls (left hemisphere: $F_{(1,30)}=4.74, p=.04$, partial $\eta^{2}=.14$; right hemisphere: $F_{(1,30)}=$ $11.15, p=.002$, partial $\eta^{2}=.27$ ) but not in amusics (left hemisphere: $F_{(1,30)}=1.03, p=.32$; right hemisphere: $\left.F_{(1,30)}=0.001, p=.98\right)$. Furthermore, the interaction between group, regularity, and anteriority was marginally significant, $F_{(1,30)}=4.10, p=.05$, partial $\eta^{2}=.12$, as the ERAN had an anterior maximum for controls (anterior regions: $F_{(1,30)}=7.68, p=.009$, partial $\eta^{2}=.20$; posterior regions: $F_{(1,30)}=5.95, p=.02$, partial $\eta^{2}=.17$ ) but not for amusics (anterior regions: $F_{(1,30)}=0.62, p=.44$; posterior regions: $F_{(1,30)}=2.77, p=.11$ ).

At approximately 450-700 msec, irregular chords also elicited a late frontal negativity (N5) and a late positive component (LPC). An ANOVA with factors of group, regularity, anteriority, and hemisphere for the time window from 450 to $700 \mathrm{msec}$ revealed an interaction between group, regularity, and anteriority, $F_{(1,30)}=4.10, p=.05$, partial $\eta^{2}=.12$, reflecting that an N5 and an LPC were elicited in controls (anterior regions: $F_{(1,30)}=9.61, p$ $=.004$, partial $\eta^{2}=.24$ for the N5; posterior regions: $F_{(1,30)}=6.89, p=.01$, partial $\eta^{2}=.19$ for the LPC) but not in amusics (anterior regions: $F_{(1,30)}=0.06, p=.81$; posterior regions: $F_{(1 \text {, }}$ $30)=1.50, p=.23)$. No other main effects or interactions related to regularity were significant (see Tables S2 and S3, Supplemental materials for more details).

Consistent with previous studies (e.g., Koelsch et al., 2007), for controls, irregular endings elicited an ERAN as compared with regular endings, followed by an N5 and an LPC at the late stage of processing. Since it has previously been suggested that the ERAN reflects 
the detection of syntactic violation and that the N5 reflects structural integration (Koelsch et al., 2007), these results indicated that controls perceived the violation of musical syntax and in turn integrated the harmony at the late processing stage. Furthermore, the observed LPC may reflect the processes of structural re-analysis or structural repair that require attention (Besson \& Schön, 2001; Koelsch \& Siebel, 2005; Patel, Gibson, Ratner, Besson, \& Holcomb, 1998). Indeed, given that the progression between the dominant and the supertonic represents a clear-cut violation that did not require holding information on-line over a delayed period, this manipulation allowed further processes that were related to structural repair to happen. For amusics, however, irregular endings did not elicit an ERAN, N5, or LPC, suggesting impairments in the detection and integration of harmonic violations. These ERP results are consistent with previous findings that amusia is associated with impaired processing of musical structures (Jiang et al., 2016; Peretz, Brattico, Järvenpää, \& Tervaniemi, 2009; Tillmann, Lalitte, Albouy, Caclin, \& Bigand, 2016; Zendel et al., 2015). Furthermore, amusics showed worse performance in rejecting irregular sequences, as compared to regular sequences. This response bias may be attributed to the rare occurrence of irregular sequences in everyday musical listening, which may make it difficult for amusics to judge irregular sequences.

\section{Experiment 2}

As explained earlier, although the processing of authentic cadence reflects the perception of harmonic syntactic structures, hierarchical syntactic relations can also arise from long-distance dependencies between the half cadence and the authentic cadence in 
paired phrases. Indeed, according to the generative theory of tonal music (Lerdahl \& Jackendoff, 1987) and the generative syntax model (Rohrmeier, 2011), the final chord of the tonic hierarchically prolongs the first chord of the sequence and closes the established dominant that remains open at the end of the half cadence. Thus, in Experiment 2, we focused on the long-distance dependency of the syntactic structure by examining whether the half cadence in the first phrase affects the processing of the authentic cadence in the second phrase in amusia.

In Western tonal music, the musical form (e.g., the sonata form) often takes a three-part arrangement of ABA (Webster, 2001). Harmonically, in the sonata form the exposition establishes the tonic key, and then, the development modifies the ideas presented in the exposition by harmonic instability. In most cases, this is brought about by fast modulations to relatively distant tonal areas (Webster, 2001). Given that the modulation of key is widely applied in the development of music events, for ecological validity, we modulated the key of the third to fifth chords of the first phrase in the irregular condition (Figure 5 and Audio Files S3 and S4), which is reminiscent of the modulation of key in the development of the sonata form. Thus, similar to the design in Koelsch et al. (2013), the final chord in our irregular condition did not prolong the first chord of the sequence, nor did it close the open dominant established by the first phrase. Unlike the design in Koelsch et al. (2013), however, we did not transpose the key of the entire first phrase, but only manipulated the half cadence in the first phrase. In this case, any effect from the final chord would only be due to the irregular long-distance dependency between the half cadence and the final chord. We recorded the neural responses to the tonic (i.e., the final chord) in the authentic cadence in the last phrase. Considering that the ERAN-like response and N5 are supposed to reflect the processing of hierarchical syntactic structures represented by long-distance dependencies (Koelsch, Rohrmeier, Torrecuso, \& Jentschke, 2013), we predicted that irregular long-distance 
dependencies would elicited an ERAN-like response and an N5 in controls but not in amusics.

Insert Figure 5, about here.

\subsection{Method}

\subsubsection{Participants}

The recruitment and inclusion criteria of the participants were identical to Experiment 1. Sixteen amusics and 16 matched controls took part in the present experiment. Only one amusic and two controls did not participate in Experiment 1. Table 2 shows the participants' demographic information, the mean scores of the MBEA, and the pitch discrimination thresholds for the two groups.

Insert Table 2, about here.

\subsubsection{Stimuli}

Two original chord sequences were created and then transposed to 12 major keys, resulting in 24 different sequences. Each harmonic sequence contained two phrases, and each phrase consisted of five chords. There were two different versions for each of the 24 sequences, in which the half cadence in the first phrase constituted either regular or irregular 
long-distance dependencies with the second phrase. In the regular version, the first phrase ended with a half cadence (i.e., on an open dominant) while the second phrase ended with an authentic cadence, in which the final chord hierarchically prolonged the first chord as well as closed the established dominant that remained open at the end of the half cadence. In the irregular version, the key of the third to fifth chords of the first phrase was modulated. By doing so, the second phrase of each irregular sequence prolonged the first chord of the sequence but did not close the open dominant established by the first phrase (see Figure 5).

That is, the long-distance dependency between the first and the second phrase was not fulfilled. This manipulation led to an irregularity of long-distance dependency, while keeping the local structure of the second phrase intact.

The final stimuli thus consisted of 48 chord sequences, of which 24 were regular and 24 were irregular. Each sequence was presented 4 times, resulting in 192 sequences for the entire experiment. Presentation time of the chords was $500 \mathrm{msec}$, except for the fifth and the final chords that lasted $1000 \mathrm{msec}$. Sequences were created using Cubase 5.1 (Steinberg Media Technologies GmbH, Hamburg, Germany), exported with the grand piano timbre (Galaxy Vienna Grand Library), with $44.1 \mathrm{kHz}$ sampling rate and 16-bit resolution. The loudness was normalized with Adobe Audition CS 6 at an approximate intensity of 68 dB SPL.

\subsubsection{Procedure}

The procedure, ERP recording, and data analysis were the same as in Experiment 1. In this experiment, an average of $15.3 \%$ of all trials were rejected, and 81 trials $(S D=9)$ were 
retained per condition, with rejections being similarly distributed across groups and conditions $(F \mathbf{S}<1)$. Experiment 1 and 2 were separated by a three-month interval.

\subsection{Results and discussion}

\subsubsection{Behavioral results}

Figure 6 shows the percentages of correct judgments $( \pm 1 S E M)$ for the regular and irregular sequences in controls and amusics, respectively. The factor regularity was entered into a repeated measures ANOVA as the within-subjects factor and group as the between-subjects factor. There was a main effect of group, $F_{(1,30)}=19.89, p<.001$, partial $\eta^{2}$ $=.40$, as controls showed higher accuracy in ir/regularity judgment $(M=71.31 \%, S D=15.62)$ than amusics $(M=52.50 \%, S D=6.09)$. The performance of amusics did not differ significantly from the chance level $\left(t_{(15)}=1.64, p=.12\right.$; one-sample $t$-test $)$. Further analysis showed that performance of amusics was above the chance level in the regular condition $\left(t_{(15)}\right.$ $=4.53, p<.001$, Cohen' $d=1.13$; one-sample $t$-test) but below the chance level in the irregular condition $\left(t_{(15)}=-4.04, p=.001\right.$, Cohen' $d=-1.01$; one-sample $t$-test $)$. Moreover, the main effect of regularity was also significant, $F_{(1,30)}=26.16, p<.001$, partial $\eta^{2}=.47$, with both groups performing better on the regular condition $(M=71.12 \%, S D=14.67)$ than the irregular condition $(M=52.78 \%, S D=21.45)$. No significant interaction between group and regularity was observed $\left(F_{(1,30)}=2.95, p=.10\right)$.

Insert Figure 6, about here. 
Bivariate correlation analysis indicated that the accuracy rates were not significantly correlated with the global MBEA scores, pitch detection thresholds, or pitch direction discrimination thresholds for either amusics or controls (all $p \mathrm{~s}>.09$ ). Furthermore, no significant correlation was found when accuracy rates in the regular and irregular conditions were considered separately, for either amusics or controls (all $p \mathrm{~s}>.35$, see Table S4, Supplemental materials for details).

\subsubsection{Electrophysiological results}

Figure 7 shows the electric brain responses to the final chords under harmonically regular and irregular chord conditions at four representative electrodes. For controls, irregular endings elicited negative potentials with an approximate onset of $110 \mathrm{msec}$ and an approximate peak latency of $250 \mathrm{msec}$. This early negativity is reminiscent of the ERAN, although it was not lateralized to the right. However, the early negative potentials were not observed in amusics. Furthermore, irregular endings also elicited fronto-central negative potentials at a late time window $(400-800 \mathrm{msec})$ in controls but not in amusics. Based on visual inspection and previous research on the ERAN and N5 (Koelsch et al., 2000; Koelsch et al., 2007), a time window from 110 to $250 \mathrm{msec}$ after the onset of the final chords was selected for the analysis of the ERAN-like response, and a time window from 400 to 800 msec was selected for the analysis of the N5.

Insert Figure 7, about here. 
An ANOVA with factors of group, regularity, anteriority, and hemisphere for the early time window $(110-250 \mathrm{msec})$ revealed an interaction between group and regularity, $F_{(1,30)}=$ 17.73, $p<.001$, partial $\eta^{2}=.37$, owing to the fact that the ERAN-like response was elicited in controls $\left(F_{(1,30)}=18.54, p<.001\right.$, partial $\left.\eta^{2}=.38\right)$ but not in amusics $\left(F_{(1,30)}=2.72, p=.11\right)$. The scalp topographies in Figure 6 suggest that the ERAN-like response had an anterior maximum, but there was no significant interaction between anteriority and regularity ( $p$ $=.09)$, nor between anteriority, regularity, and group $(p=.72)$.

At approximately 400-800 msec, irregular chords also elicited a frontal negativity. An ANOVA with factors of group, regularity, anteriority, and hemisphere for the time window from 400 to $800 \mathrm{msec}$ indicated an interaction between group and regularity, $F_{(1,30)}=4.40, p$ $=.04$, partial $\eta^{2}=.13$, as an N5 was elicited in controls $\left(F_{(1,30)}=8.35, p=.007\right.$, partial $\eta^{2}$ $=.22)$ but not in amusics $(F<1)$. The scalp topographies in Figure 6 suggest that the N5 had an anterior maximum, but there was no significant interaction between anteriority and regularity $(p=.09)$, nor between anteriority, regularity, and group $(p=.16)$. No other main effects or interactions related to regularity were significant (see Table S5, Supplemental materials for details).

To examine the effect of local transition probability between the last chord of the first phrase and the first chord of the second phrase, we analyzed the ERPs of the first chord of the second phrase. The results showed that an early negativity over the frontal scalp was elicited in controls but not in amusics. A global ANOVA for a time window from 100-200 
msec indicated an interaction between group, regularity, and anteriority, $F_{(1,30)}=4.17, p$ $=.05$, partial $\eta^{2}=.12$, owing to the fact that the early negativity with a frontal distribution was elicited in controls (anterior regions: $F_{(1,30)}=5.79, p=.02$, partial $\eta^{2}=.16$; posterior regions: $F_{(1,30)}=3.32, p=.08$ ) but not in amusics (anterior regions: $F_{(1,30)}=1.40, p=.25$; posterior regions: $F_{(1,30)}=0.82, p=.37$ ) (see Supplemental materials, Table S6, First chord of the second phrase for more details).

To rule out the possibility that the effect observed in the ending chord was due to the local transitions from the first phrase to the second phrase, we also compared the ERPs of the second chord and the penultimate chord of the second phrase between regular and irregular conditions. For the second chord of the second phrase, the results did not show any significant effect related to regularity (for statistics see Supplemental materials, Table S6, Prefinal tonic). Similarly, no significant effect related to regularity was observed for the penultimate chord (for statistics see Supplemental materials, Table S6, Penultimate chord). These findings ruled out the possibility that the ERP effects elicited by the final chords were due to sensory factors.

In summary, syntactic processing elicited an ERAN-like response and an N5 in the control participants, indicating that controls perceived harmonic syntactic structures induced by the violations of long-distance dependencies. This finding is consistent with the results by Koelsch et al. (2013), who also found an ERAN-like response and an N5 in the processing of long-distance syntactic dependency in nonmusicians. However, amusics failed to elicit these ERP responses. The absence of electrophysiological effects was consistent with the poor 
performance of amusics at the behavioral level, suggesting that individuals with amusia could not detect the violations of long-distance dependencies, nor could they integrate the harmonic information at the late stage of processing. Compared with the behavioral results in Experiment 1, however, both controls and amusics showed worse performance in the rejection of irregular sequences. Although such a response bias between regular and irregular conditions has been reported for typical people (e.g., James et al., 2008; Koelsch et al., 2007), this finding suggests that irregular long-distance dependencies are more difficult to detect than irregular local dependencies.

\subsubsection{Correlation of ERPs between the two experiments}

To test for the correlations between the ERP effects elicited by local and long-distance dependencies of syntactic structures, we conducted additional correlation analyses between the two experiments for the ERAN and N5, respectively. Only the data from the same participants (15 amusics and 14 controls) were considered. Results showed that the ERAN in Experiment 1 was not significantly correlated with the ERAN-like response in Experiment 2 for either amusics or controls ( $p$ s $>.19)$. However, the N5 in Experiment 1 was significantly correlated with that in Experiment 2 for controls $\left(r_{(13)}=.70, p<.01\right)$, although there was no significant correlation for amusics $(p>.26)$. These findings suggest that the ERPs elicited by local and long-distance dependencies of harmonic syntactic structures were similar at the late stage of processing for control participants. 


\section{General discussion}

Hierarchical syntactic relations in music can arise from local harmonic structures in an authentic cadence, or from long-distance dependency syntactic structures resulted from the relation between a half cadence and an authentic cadence in paired phrases. The present study used ERPs to investigate the impact of amusia on the neural processing of harmonic syntactic structures induced by local (Experiment 1) and long-distance dependencies (Experiment 2). In Experiment 1, by manipulating the ending chord of the authentic cadence in harmonic sequences, we observed that irregular endings elicited an ERAN followed by an N5 and an LPC in controls but not in amusics, as compared with regular endings. This finding indicates that amusics could not process local syntactic dependencies in the authentic cadence. In Experiment 2, by manipulating the hierarchical syntactic relation between the half cadence and the authentic cadence, we revealed that irregular long-distance dependencies elicited an ERAN-like effect and an N5 in controls but not in amusics. This finding suggests that amusics were also impaired in perceiving syntactic structures of long-distance dependencies in music. In both experiments, the ERP responses reflected the performance on the behavioral tasks for both amusics and controls. Together, our findings suggest that amusics are impaired in the early detection of the violations of hierarchical syntactic structures, and in the subsequent structural integration and meaning processing.

The main contribution of the current study was to show the impact of amusia on the neural processing of harmonic syntactic structures. Even without musical training, normal listeners can develop sensitivity to hierarchical syntactic structures in music based on local 
(see Bigand \& Poulin-Charronnat, 2006 for a review) and long-distance dependencies (Koelsch et al., 2013) through musical exposure and perceptual learning. However, amusics in the present study were unable to process syntactic structures based on local and long-distance dependencies. These results confirm the previous finding that amusics cannot explicitly process musical structure (Jiang et al., 2016; Peretz et al., 2009; Tillmann, Lalitte, et al., 2016; Zendel et al., 2015), and extend to the processing of long-distance dependency of syntactic structures.

The impairments in the processing of harmonic syntactic structures in amusics might be related to their reduced sensitivity to tonality. Indeed, musical structure is governed by functional regularity, which is based on the hierarchy of tonal stability (see Bigand, 1993 for a review). It has been demonstrated that amusics are unable to perceive the stability of tones in a hierarchical manner (Jiang et al., 2016), and that they cannot use tonality-related cues (e.g., mode) to perceive musical tension (Jiang, Liu, \& Wong, 2017). Consequently, amusics are unable to make an explicit judgment of the tonal regularity of chords, thus resulting in their reduced sensitivity to the distinction between regular and irregular syntactic structures.

It is possible that amusics' deficits in harmonic syntactic processing are not due to their impairments in low-level music processing or pitch discrimination, given that their scores on the MBEA and their pitch perceptual thresholds were not correlated with their performance on the judgment of musical expectancy. Previous studies have also suggested that deficits in pitch perception are not related to pitch expectations (Omigie et al., 2012), musical syntax and tonality performance (Jiang et al., 2016), or musical meaning processing (Zhou, Liu, Jing, 
\& Jiang, 2017) in amusia. Our findings thus imply that the processing of harmonic syntactic structures may be cognitively and neurally distinct from mechanisms responsible for low level pitch discrimination. Taken together, amusics' deficits may be relevant to pitch processing at both low and high levels of music processing, and there may be a neural network that is specific to pitch processing in music (Norman-Haignere et al., 2016; Zhou et al., 2017). Given previous conclusions that amusics have internalized syntactic-like functions of pitches (e.g., Tillmann et al., 2012, 2016a; Zendal et al., 2015), our findings support the idea that amusics are unable to consciously access pitch information that is encoded in their auditory cortex. Indeed, it has been suggested that an alteration in consciousness can give rise to severe cognitive impairments in music (Zendal et al., 2015). The present findings thus indicate that cognitive impairments in harmonic syntactic structures might be due to deficits in conscious access of pitch.

For controls, our results provided evidence for the similarities and differences between the processing of local and long-distance dependencies of syntactic structures. Unlike the ERAN in Experiment 1, however, the ERAN-like response in Experiment 2 was not anterior-right distributed but with a slight (statistically nonsignificant) left hemispheric weighting. The ERAN and the ERAN-like response might reflect the early detection of syntactic violations induced by local and long-distance dependencies, respectively. Indeed, as revealed by our correlation analysis, the ERAN and the ERAN-like response in the two experiments was not correlated with each other. This might be because in Experiment 2 
additional syntactic processing is required to recognize the long-distance structural dependency between non-adjacent structures.

Unlike the ERAN, the amplitudes of the N5 in the two experiments were significantly correlated for controls, suggesting similar processing of the syntactic structures between the two experiments. It has been suggested that the N5 evoked by final chords probably reflects the increased amount of harmonic integration (Koelsch et al., 2000; Koelsch et al., 2013) or the processing of meaning information (Koelsch, 2011, 2012; Steinbeis \& Koelsch, 2008). Indeed, in both experiments, participants had to harmonically integrate the final chords into the previous musical contexts. Such integration might correlate with the expectancy of structural relations of musical events, which can, in turn, have meaning for the individual (Huron, 2008; Koelsch, 2012; Meyer, 1956). Therefore, our findings suggest that the processing of hierarchical syntactic structures induced by local and long-distance dependencies might be similar during the processing stage of harmonic integration or meaning processing. Future studies using neuroimaging techniques with high spatial resolution would be needed to explore the neural correlations between local and long-distance structural dependencies in music.

Hierarchical syntax is a key feature in both musical and linguistic structures. Consistent with the findings in Koelsch et al. (2013), the present study confirms that typical people are capable of perceiving hierarchical syntax in music. Moreover, our study extends previous research to reveal the intricate mechanisms of local and long-distance dependencies in hierarchical syntactic processing. Since previous linguistic-syntactic studies also showed 
neural differences between local and long-distance dependencies (e.g., Friederici, 2004; Jiang \& Zhou, 2009; Phillips, Kazanina, \& Abada, 2005), the present study provides further evidence for the "Shared Syntactic Integration Resource Hypothesis" (SSIRH) (Patel, 2003, 2008), which suggests that music and language may share cognitive and neural resources in syntactic processing.

Finally, it has been suggested that amusia is associated with a short-term pitch memory deficit (see Tillmann et al., 2016b for a review). However, pitch perception and memory are interwoven in the processing of music (Cook, 1987; Huron \& Parncutt, 1993; Jiang et al., 2013). Even if there is a possible pitch memory deficit in amusia, it is unlikely that our observed effects were due to the possible difference in memory for musical material between the two groups. Specifically, although the processing of long-distance structural dependencies may require additional working memory in establishing and maintaining syntactic representations than local dependencies (Koelsch et al., 2013), studies on linguistic syntactic processing have shown that on-line construction of syntactic form is not influenced by the capacity of the working memory (Waters \& Caplan, 2004), and that the processing of syntactic structures is neuroanatomically separate from non-syntactic working memory (Makuuchi et al., 2009). This might be due to the fact that syntactic processing in both music and language relies on the representations of syntactic regularities that already exist in a long-term memory format (Koelsch, Schmidt, \& Kansok, 2002). On the other hand, if short-term pitch memory is involved in syntactic processing and the processing of long-distance structural dependencies demands more short-term memory resources than that 
of local dependencies, then the observed effects of group difference would be different across the two experiments of our study. However, the effect sizes of the main effect of group were similar in the two experiments (0.39 in Experiment 1 and 0.40 in Experiment 2). Taken together, the observed group effects in our present study were unlikely to be due to the possible difference in memory for musical material between the two groups.

To conclude, our findings revealed that amusics were impaired in the detection of syntactic violations and subsequent harmonic integration. The amusic deficits in the processing of local and long-distance dependencies of harmonic syntactic structures might be due to their reduced sensitivity to tonality. These findings provide evidence to support the view that there may be a neural network specific to pitch processing in music, and reveal the similarities and differences between the processing of local and long-distance dependencies of musical structures. 


\section{References}

Albouy, P., Schulze, K., Caclin, A., \& Tillmann, B. (2013). Does tonality boost short-term memory in congenital amusia? Brain research, 1537, 224-232.

Alain, C., Achim, A., \& Woods, D. L. (1999). Separate memory-related processing for auditory frequency and patterns. Psychophysiology, 36(6), 737-744.

Ayotte, J., Peretz, I., \& Hyde, K. (2002). Congenital amusia : A group study of adults afflicted with a music-specific disorder. Brain, 125(2), 238-251. doi:10.1093/brain/awf028

Besson, M., \& Schön, D. (2001). Comparison between language and music. Annals of the New York Academy of sciences, 930(1), 232-258.

Bharucha, J. \& Krumhansl, C. L. (1983). The representation of harmonic structure in music: Hierarchies of stability as a function of context. Cognition, 13, 63-102.

Bigand, E. (1993). Contribution of music to research on human auditory cognition. In S. McAdams \& E. Bigand (Eds.), Thinking in sound: The cognitive psychology of human audition (pp. 231-277). Oxford: Clarendon Press.

Bigand, E., Delbé, C., Poulin-Charronnat, B., Leman, M., \& Tillmann, B. (2014). Empirical evidence for musical syntax processing? Computer simulations reveal the contribution of auditory short-term memory. Frontiers in Systems Neuroscience, 8, 94. 
Bigand, E., Poulin, B., Tillmann, B., Madurell, F., D'Adamo, D. A. (2003). Sensory versus cognitive components in harmonic priming. Journal of Experimental Psychology: Human Perception \& Performance, 29(1), 159-171.

Bigand, E., \& Poulin-Charronnat, B. (2006). Are we "experienced listeners"? A review of the musical capacities that do not depend on formal musical training. Cognition, 100, 100130.

Cook, N., (19870. The perception of large-scale tonal closure. Music Perception, 5(2),197205

Fitch, W.T., \& Hauser, M.D. (2004) Computational constraints on syntactic processing in a nonhuman primate. Science, 303(5656), 377-380.

Foxton, J. M., Dean, J. L., Gee, R., Peretz, I., \& Griffiths, T. D. (2004). Characterization of deficits in pitch perception underlying 'tone deafness'. Brain, 127(4), 801-810.

Friederici AD (2004) Processing local transitions versus long-distance syntactic hierarchies. Trends in Cognitive Sciences, 8, 245-247.

Friederici, A.D., Bahlmann, J., Heim, S., Schubotz, R.I., \& Anwander, A. (2006) The brain differentiates human and non-human grammars: Functional localization and structural connectivity. Proceedings of the National Academy of Sciences, 103(7), 2458-2463.

Hauser, M.D., Chomsky, N., \& Fitch, W.T. (2002) The faculty of language: What is it, who has it, and how did it evolve? Science, 298(5598), 1569-1579. 
Henry, M. J., \& McAuley, J. D. (2013). Failure to apply signal detection theory to the Montreal Battery of Evaluation of Amusia may misdiagnose amusia. Music Perception, 30(5), 480-496. doi:10.1525/mp.2013.30.5.480

Huron, D. (2008). Sweet anticipation: Music and the psychology of expectation. Cambridge, MA: MIT Press.

Huron, D., Parncutt, R., (1993). An improved model of tonality perception incorporating pitch salience and echoic memory. Psychomusicology, 12(2), 154-171.

Hyde, K. L., \& Peretz, I. (2004). Brains that are out of tune but in time. Psychological Science, 15(5), 356-360. doi:10.1111/j.0956-7976.2004.00683.x

Jentschke, S., Friederici, A. D., \& Koelsch, S. (2014). Neural correlates of music-syntactic processing in two-year old children. Developmental cognitive neuroscience, 9, 200-208.

Jentschke, S., \& Koelsch, S. (2009). Musical training modulates the development of syntax processing in children. Neuroimage, 47(2), 735-744.

Jiang, C., Hamm, J. P., Lim, V. K., Kirk, I. J., \& Yang, Y. (2010). Processing melodic contour and speech intonation in congenital amusics with Mandarin Chinese. Neuropsychologia, 48(9), 2630-2639. doi:10.1016/j.neuropsychologia.2010.05.009

Jiang, C., Hamm, J. P., Lim, V. K., Kirk, I. J., \& Yang, Y. (2011). Fine-grained pitch discrimination in congenital amusics with Mandarin Chinese. Music Perception, 28(5), 519-526. doi:10.1525/mp.2011.28.5.519 
Jiang, C., Lim, V.K., Wang, H., Hamm, J.P., (2013). Difficulties with pitch discrimination influences pitch memory performance: Evidence from congenital amusia. PLoS ONE, 8(10), e79216.

Jiang, C., Liu, F., \& Thompson, W. F. (2016). Impaired explicit processing of musical syntax and tonality in a group of Mandarin-speaking congenital amusics. Music Perception, $33(4), 401-413$.

Jiang, C., Liu, F., \& Wong, P. C. M. (2017). Sensitivity to musical emotion is influenced by tonal structure in congenital amusia. Scientific Reports, 7, 7624.

Jiang, X., \& Zhou, X. (2009). Processing different levels of syntactic hierarchy: An ERP study on Chinese. Neuropsychologia, 47(5), 1282-1293.

Koelsch, S. (2009). Music-syntactic processing and auditory memory: Similarities and differences between ERAN and MMN. Psychophysiology, 46(1), 179-190.

Koelsch, S. (2011). Towards a neural basis of processing musical semantics. Physics of Life Reviews, 8(2), 89-105. doi:10.1016/j.plrev.2011.04.004

Koelsch, S. (2012). Brain and music. Oxford, UK: Wiley-Blackwell.

Koelsch, S., Gunter, T., Friederici, A. D., \& Schröger, E. (2000). Brain indices of music processing:"non-musicians" are musical. Journal of cognitive neuroscience, 12(3), 520541.

Koelsch, S., \& Jentschke, S. (2010). Differences in electric brain responses to melodies and chords. Journal of cognitive neuroscience, 22(10), 2251-2262. 
Koelsch, S., Jentschke, S., Sammler, D., \& Mietchen, D. (2007). Untangling syntactic and sensory processing: An ERP study of music perception. Psychophysiology, 44(3), 476490.

Koelsch, S., Rohrmeier, M., Torrecuso, R., \& Jentschke, S. (2013). Processing of hierarchical syntactic structure in music. Proceedings of the National Academy of Sciences, 110(38), 15443-15448. doi:10.1073/pnas.1300272110

Koelsch, S., Schmidt, B.-H. \& Kansok, J. (2002). Effects of musical expertise on the early right anterior negativity: An event-related brain potential study. Psychophysiology, 39, $657-663$.

Koelsch, S., \& Siebel, W. A. (2005). Towards a neural basis of music perception. Trends in cognitive sciences, 9(12), 578-584.

Kolk, H. H. J., Chwilla, D. J., van Herten, M., \& Oor, P. J. W. (2003). Structure and limited capacity in verbal working memory: A study with event-related potentials. Brain and Language, 85(1), 1-36.

Krumhansl, C. L. (1990). Cognitive foundations of musical pitch. New York, NY: Oxford University Press.

Lerdahl, F., \& Jackendoff, R. (1983). A generative theory of tonal music. Cambridge,MA: MIT Press.

Liu, F., Maggu, A. R., Lau, J. C., \& Wong, P. C. (2015). Brainstem encoding of speech and musical stimuli in congenital amusia: evidence from Cantonese speakers. Frontiers in Human Neuroscience, 8, 1029. doi:10.3389/fnhum.2014.01029 
Liu, F., Patel, A. D., Fourcin, A., \& Stewart, L. (2010). Intonation processing in congenital amusia: Discrimination, identification and imitation. Brain, 133(6), 1682-1693. doi:10.1093/brain/awq089

Loui, P., Grent-'t Jong, T., Torpey, D., \& Woldorff, M. (2005). Effects of attention on the neural processing of harmonic syntaxin Western music. Cognitive Brain Research, 25, $678-687$.

Makuuchi, M., Bahlmann, J., Anwander, A. \& Friederici, A. D. (2009). Segregating the core computational faculty of human language from working memory. Proceedings of the National Academy of Sciences, 106, 8362-8367.

Meyer, L. B. (1956). Emotion and meaning in music. Chicago: University of Chicago Press.

Narmour, E. (1983). Some major theoretical problems concerning the concept of hierarchy in the analysis of tonal music. Music Perception: An Interdisciplinary Journal, 1(2), 129199.

Norman-Haignere, S. V., Albouy, P., Caclin, A., McDermott, J. H., Kanwisher, N. G., \& Tillmann, B. (2016). Pitch-responsive cortical regions in congenital amusia. The Journal of Neuroscience, 36(10), 2986-2994. doi:10.1523/JNEUROSCI.2705-15.2016

Oldfield, R. C. (1971). The assessment and analysis of handedness: The Edinburgh inventory. Neuropsychologia, 9(1), 97-113. doi:10.1016/0028-3932(71)90067-4

Omigie, D., Pearce, M. T., \& Stewart, L. (2012). Tracking of pitch probabilities in congenital amusia. Neuropsychologia, 50(7), 1483-1493. 
Patel, A. D. (2003). Language, music, syntax and the brain, Nature Neuroscience, 6(7): 674681.

Patel, A. D. (2008). Music, language, and the brain. NewYork, NY: Oxford University Press.

Patel, A. D., Gibson, E., Ratner, J., Besson, M., \& Holcomb, P. J. (1998). Processing syntactic relations in language and music: An event-related potential study. Journal of cognitive neuroscience, 10(6), 717-733.

Peretz, I., Ayotte, J., Zatorre, R. J., Mehler, J., Ahad, P., Penhune, V. B., \& Jutras, B. (2002). Congenital amusia: A disorder of fine-grained pitch discrimination. Neuron, 33(2), 185191. doi:10.1016/S0896-6273(01)00580-3

Peretz, I., Brattico, E., Järvenpää, M., \& Tervaniemi, M. (2009). The amusic brain: In tune, out of key, and unaware. Brain, 132(5), 1277-1286. doi:10.1093/brain/awp055

Peretz, I., Champod, A. S., \& Hyde, K. L. (2003). Varieties of musical disorders: The Montreal Battery of Evaluation of Amusia. Annals of the New York Academy of sciences, $999,58-75$.

Pfeifer, J., \& Hamann, S. (2015). Revising the diagnosis of congenital amusia with the Montreal Battery of Evaluation of Amusia. Frontiers in Human Neuroscience, 9, 161. doi:10.3389/fnhum.2015.00161

Phillips, C., Kazanina, N., \& Abada, S. H. (2005). ERP effects of the processing of syntactic long-distance dependencies. Cognitive Brain Research, 22(3), 407-428. 
Poulin-Charronnat, B., Bigand, E., \& Koelsch, S. (2006). Processing of musical syntax tonic versus subdominant: An event-related potential study. Journal of Cognitive Neuroscience, 18(9), 1545-1554.

Rohrmeier, M. (2011). Towards a generative syntax of tonal harmony. Journal of Mathematics and Music, 5(1), 35-53.

Rosner, B. S., \& Narmour, E. (1992). Harmonic closure: Music theory and perception. Music Perception: An Interdisciplinary Journal, 9(4), 383-411.

Steinbeis, N., \& Koelsch, S. (2008). Shared neural resources between music and language indicate semantic processing of musical tension-resolution patterns. Cerebral Cortex, 18(5), 1169-1178. doi:10.1093/cercor/bhm149

Sun, L., Liu, F., Zhou, L., \& Jiang, C. (2018). Musical training modulates the early but not the late stage of rhythmic syntactic processing. Psychophysiology, 55(2), e12983.

Sun, Y., Lu, X., Ho, H. T., Johnson, B. W., Sammler, D., \& Thompson, W. F. (2018). Syntactic processing in music and language: Parallel abnormalities observed in congenital amusia. NeuroImage: Clinical, 19, 640-651.

Tervaniemi, M., Rytkönen, M., Schröger, E., Ilmoniemi, R. J., Näätänen, R. J. L., \& Memory. (2001). Superior formation of cortical memory traces for melodic patterns in musicians. Learning and Memory, 8(5), 295-300.

Tillmann, B., Bigand, E., \& Madurell, F. (1998). Local versus global processing of harmonic cadences in the solution of musical puzzles. Psychological Research, 61(3), 157-174. 
Tillmann, B., Gosselin, N., Bigand, E., \& Peretz, I. (2012). Priming paradigm reveals harmonic structure processing in congenital amusia. Cortex, 48(8), 1073-1078. doi:10.1016/j.cortex.2012.01.001

Tillmann, B., Lalitte, P., Albouy, P., Caclin, A., \& Bigand, E. (2016a). Discrimination of tonal and atonal music in congenital amusia: The advantage of implicit tasks. Neuropsychologia, 85, 10-18. doi:10.1016/j.neuropsychologia.2016.02.027

Tillmann, B., Lévêque, Y., Fornoni, L., Albouy, P., \& Caclin, A. (2016b). Impaired short-term memory for pitch in congenital amusia. Brain research, 1640, 251-263. doi:10.1016/j.brainres.2015.10.035

Vaughan-Evans, A., Kuipers, J. R., Thierry, G., \& Jones, M. W. (2014). Anomalous transfer of syntax between languages. Journal of Neuroscience, 34(24), 8333-8335.

Waters, G. S. \& Caplan, D. (2004). Verbal working memory and on-line syntactic processing: Evidence from self-paced listening. The Quarterly Journal of Experimental Psychology, 57, 129-163.

Webster, J. (2001). Sonata form. In S. Sadie (Ed.), New Grove dictionary of music and musicians (2 ed., Vol. 23, pp. 287-701).

Williamson, V. J., Baddeley, A. D., \& Hitch, G. J. (2010). Musicians' and nonmusicians' short-term memory for verbal and musical sequences: Comparing phonological similarity and pitch proximity. Memory\& Cognition, 38(2), 163-175. 
Zendel, B. R., Lagrois, M.-É., Robitaille, N., \& Peretz, I. (2015). Attending to pitch information inhibits processing of pitch information: The curious case of amusia. The Journal of Neuroscience, 35(9), 3815-3824. doi:10.1523/JNEUROSCI.3766-14.2015

Zhou, L., Liu, F., Jing, X., \& Jiang, C. (2017). Neural differences between the processing of musical meaning conveyed by direction of pitch change and natural music in congenital amusia. Neuropsychologia, 96, 29-38. 


\section{Acknowledgments}

This work was supported by the National Natural Science Foundation of China (Grant No. 31470972 to C. J. and F. L., and Grant No. 31500876 to L. Z.), and the European Research Council Starting Grant to F. L. and C. J. (CAASD, No. 678733). We wish to thank the editor, Dr. Natalie Phillips, and three anonymous reviewers for their insightful comments and helpful suggestions on our earlier draft. 


\section{Figure captions}

Figure 1. Hierarchical syntactic structures in paired phrases. The first phrase ends on an open dominant $(\mathrm{V})$, and the second phrase ends on a tonic (I). The tree structure above the scores represents a schematic diagram of the structural dependencies. In the second phrase, the penultimate chord (V) is followed by the resolving tonic (I), representing the local harmonic dependency in the authentic cadence. In the first phrase, the open dominant (V) in the half cadence is not immediately followed by a resolving tonic chord but implies its resolution with the final tonic (I), representing the long-distance dependency between the half cadence and the final tonic. The same dependency exists between the initial and final tonic.

Figure 2. Illustration of stimuli in Experiment 1. (A) Regular version. The first phrase ends on an open dominant, and the second phrase ends on a tonic. The penultimate chord (V) is immediately followed by the resolving tonic (I), representing the local harmonic dependency in the authentic cadence. Moreover, the final tonic prolongs the first chord of the sequence and closes the established dominant that remained open at the end of the half cadence. (B) Irregular version. The supertonic chord was used as an irregular ending, representing the violation of the local harmonic dependency in the authentic cadence. Moreover, the supertonic ending does not prolong the first chord of the sequence or close the established dominant at the end of the half cadence. However, the use of the supertonic does not introduce new chord function, and the frequency of occurrence of the ending chords has been controlled for between the regular and irregular conditions. The arrows indicate new 
pitches that were not contained in any of the previous chords: the final tonic contained two new pitches (in both the top voice and the base voice), whereas the final supertonic contained one new pitch (in the top voice). Therefore, any effect responded to the syntactically irregular condition should not be due to the factor of sensory novelty, because the final tonics contained more new pitches than the final supertonics. Participants were instructed to decide whether the progression of the chords was consistent with their expectation after the presentation of each chord sequence.

Figure 3. Experiment 1: Boxplots of accuracy rates in amusics and controls for the judgment of musical completion under regular and irregular conditions. These boxplots contain the extreme of the lower whisker, the lower hinge, the median, the upper hinge, and the extreme of the upper whisker. The two hinges are the first and third quartile, and the whiskers extend to the most extreme data. The black crosses denote arithmetic mean. ${ }^{*} p$ $<.05 ; * * * p<.001 ;$ n.s., no significant difference.

Figure 4. Experiment 1: Grand mean waveforms elicited by regular and irregular ending chords at four representative electrode sites for amusics and controls, respectively. Bottom: Scalp distribution of the irregular-minus-regular difference waves in the 110-230 msec and 450-700 msec latency ranges for amusics and controls, respectively.

Figure 5. Illustration of stimuli in Experiment 2. (A) Regular version. The first phrase ends on an open dominant, and the second phrase ends on a tonic. The final tonic (I) prolongs the first chord of the sequence (I) and closes the established dominant (V) that remained open at the end of the half cadence, representing the long-distance dependencies of musical 
syntactic structures. (B) Irregular version. In the first phrase, the key of the last three chords was modulated. In this case, the final chord in the irregular condition prolongs the first chord of the sequence whereas it does not close the established dominant that remained open at the end of the half cadence, representing the violation of the long-distance dependency of syntactic structure. Moreover, the local dependency between the penultimate chord (V) and the final tonic (I) keeps intact. Participants were instructed to decide whether the progression of the chords was consistent with their expectation after the presentation of each chord sequence.

Figure 6. Experiment 2: Boxplots of accuracy rates in amusics and controls for the judgment of musical completion under regular and irregular conditions. ${ }^{*} p<.05 ;{ }^{* * *} p$ $<.001$

Figure 7. Experiment 2: Grand mean waveforms elicited by regular and irregular ending chords at four representative electrode sites for amusics and controls, respectively. Bottom: Scalp distribution of the irregular-minus-regular difference waves in the 110-250 msec and 400-800 msec latency ranges for amusics and controls, respectively. 
Figure 1

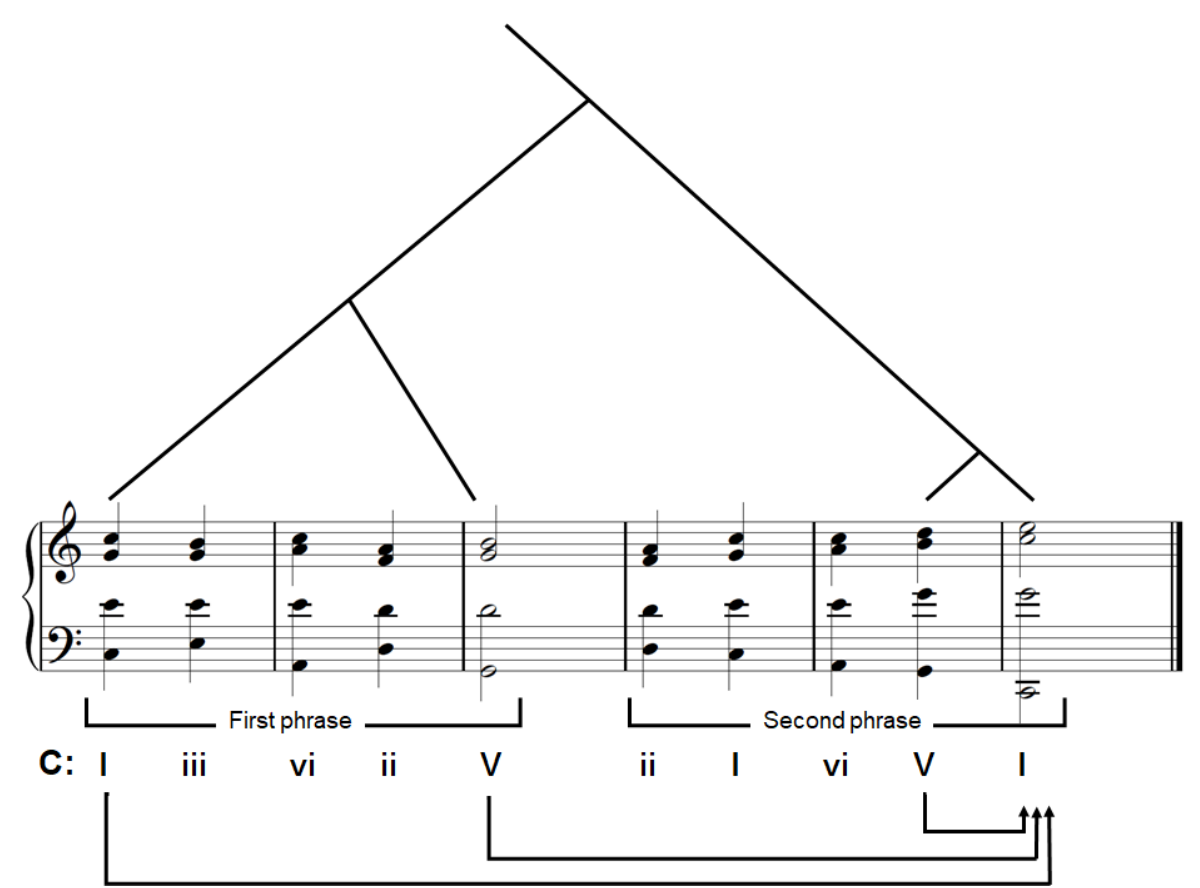


Figure 2

(A)

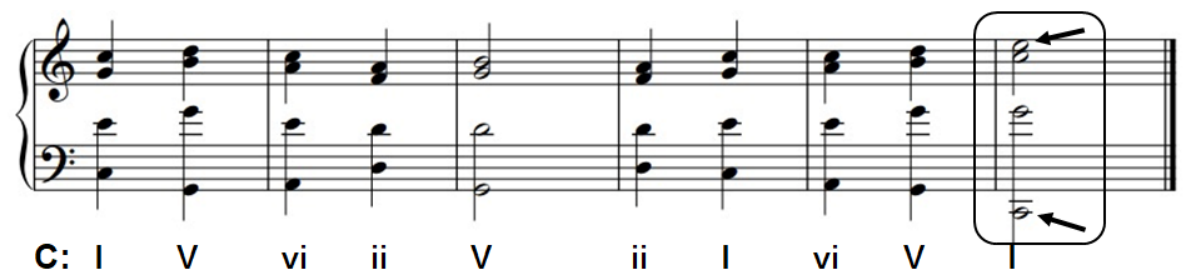

C: I v vi ii

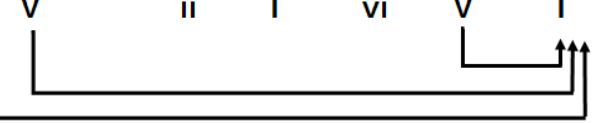

(B)

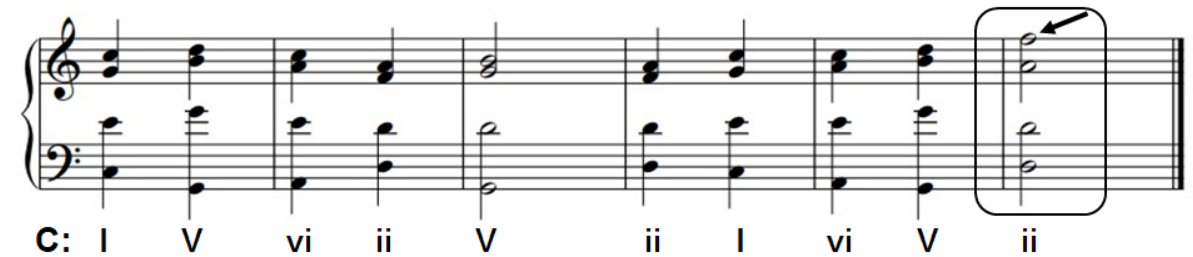

C:

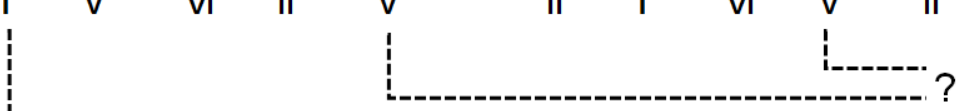


Figure 3

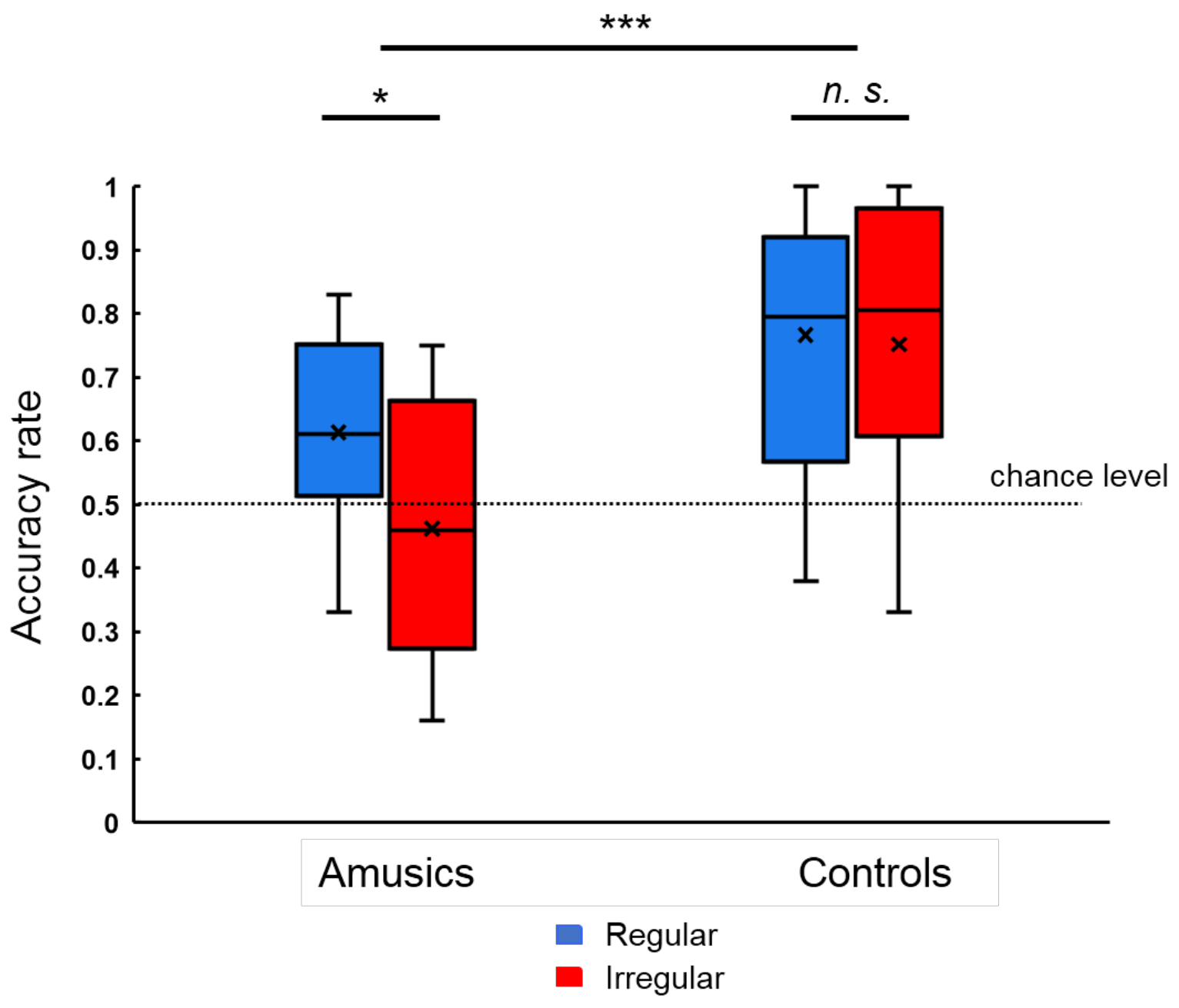


Figure 4

\section{Amusics}
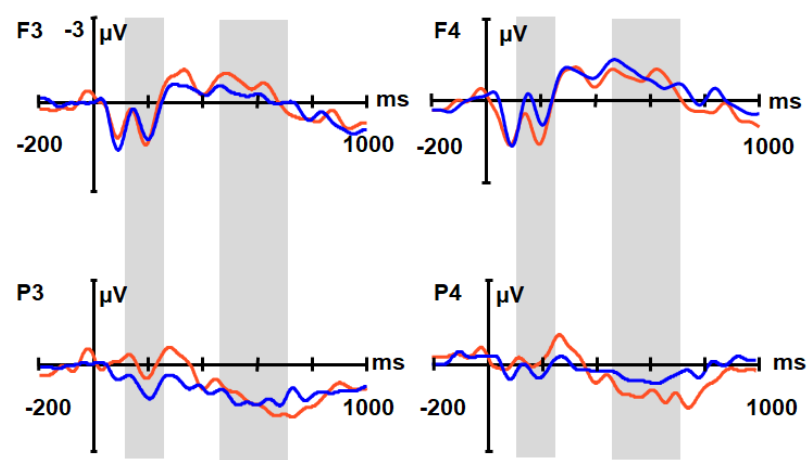

Regular

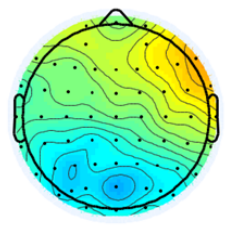

110-230 ms

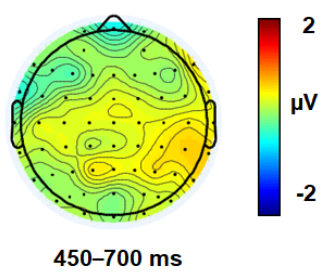

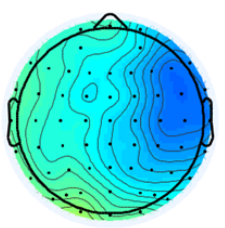

110-230 ms
Controls
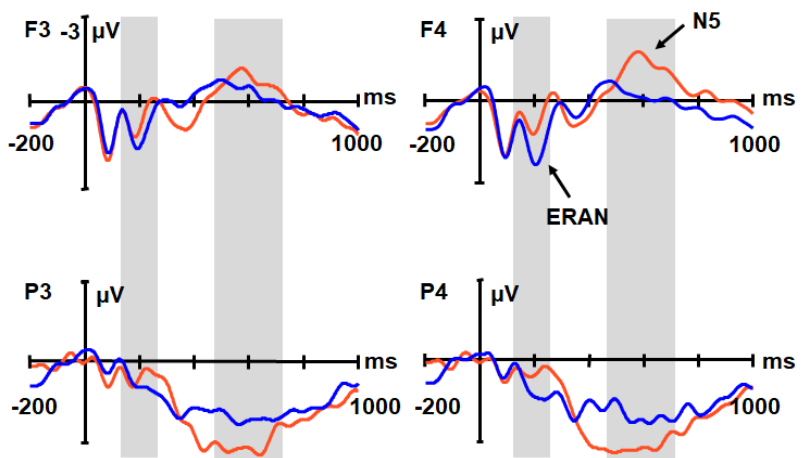

Irregular
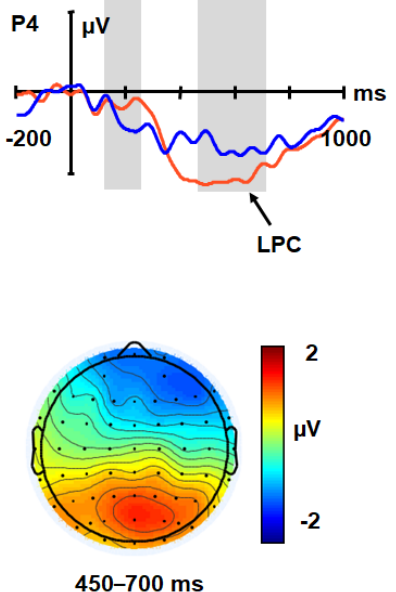
Figure 5

(A)

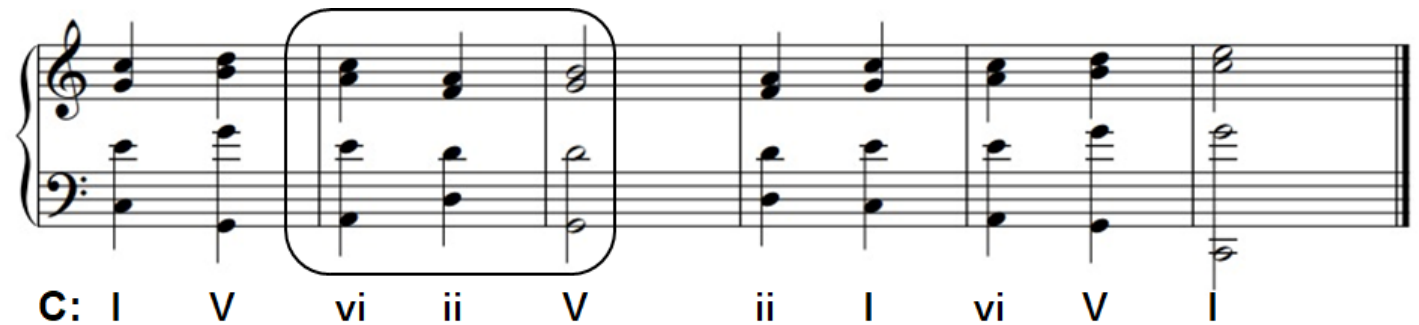

(B)

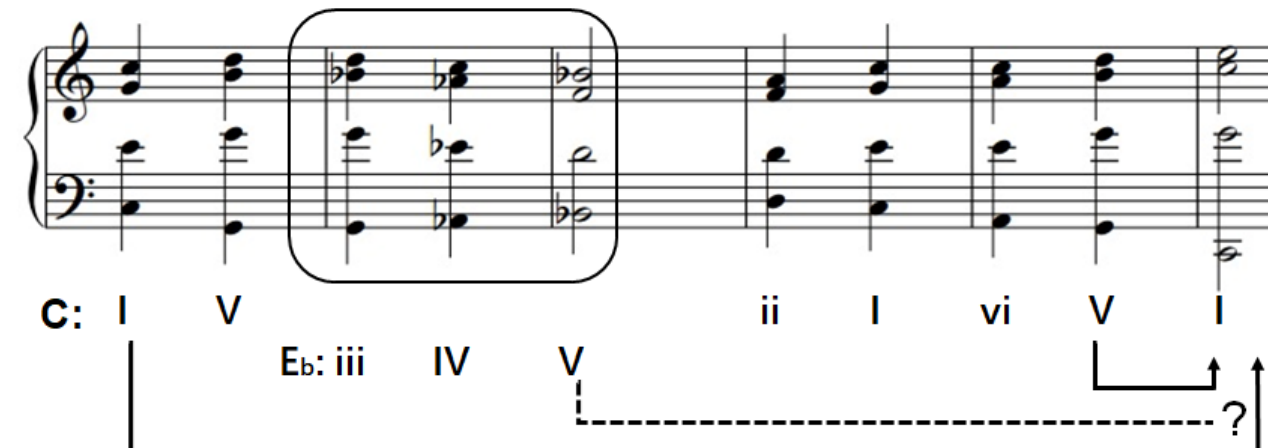


Figure 6

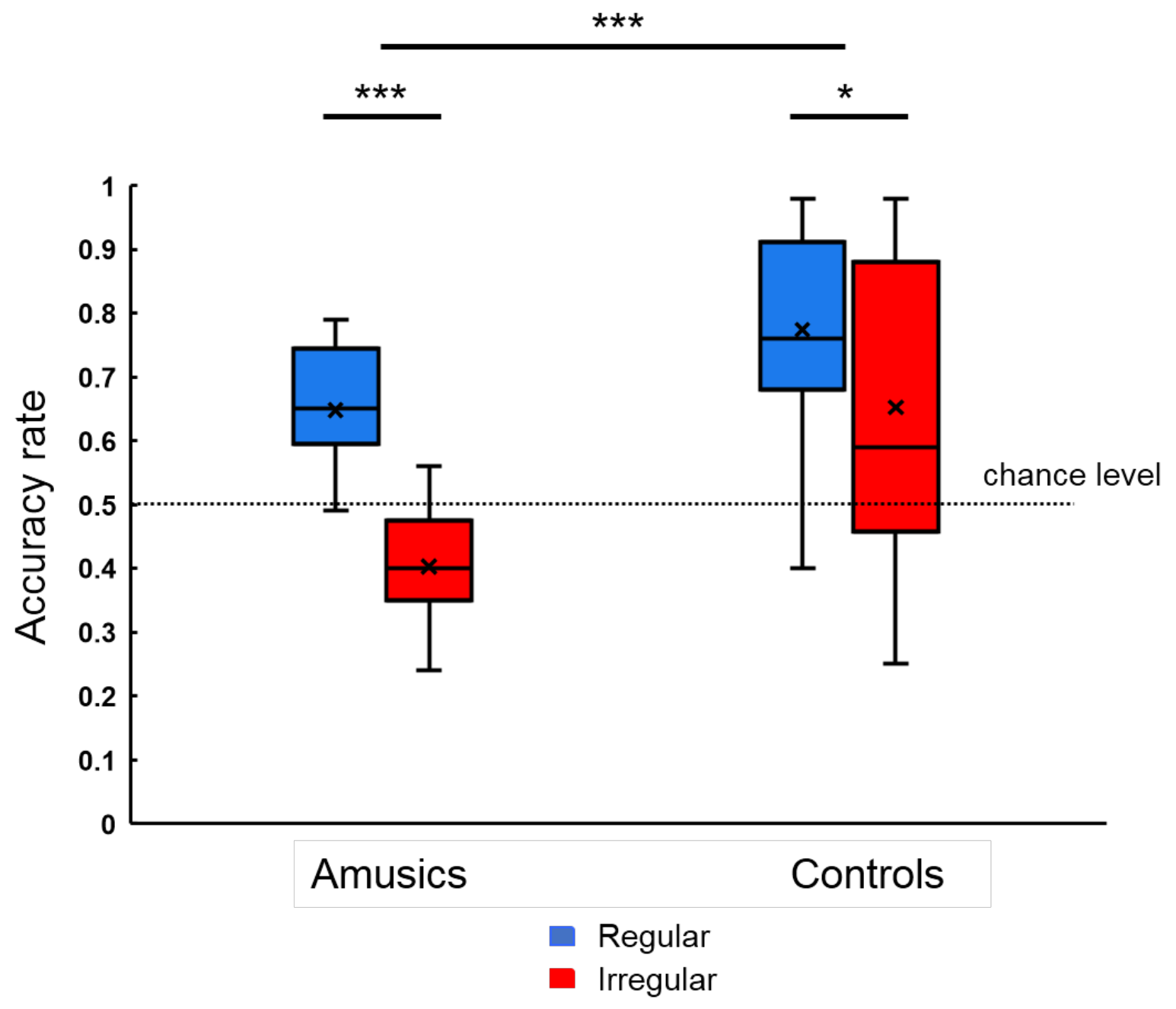




\section{Figure 7}

\section{Amusics}
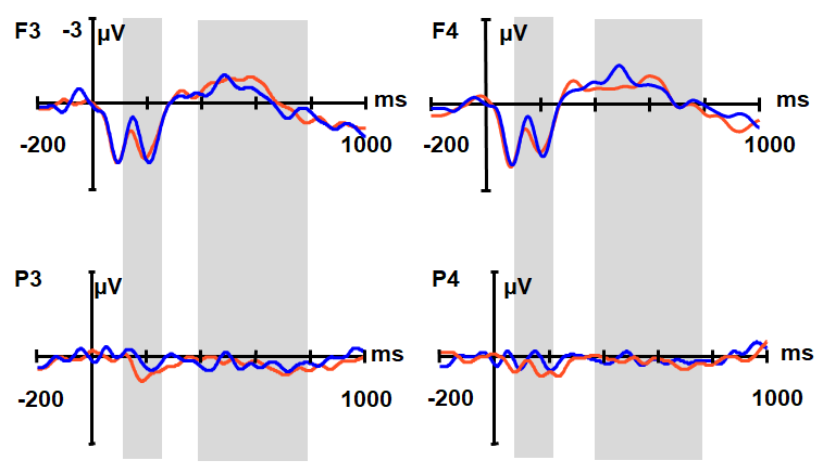

Regular

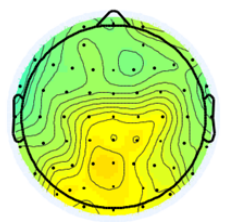

$110-250 \mathrm{~ms}$

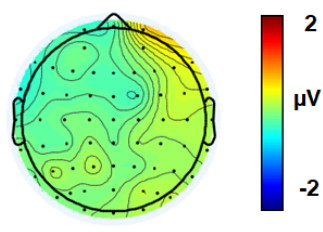

400-800 ms

\section{Controls}

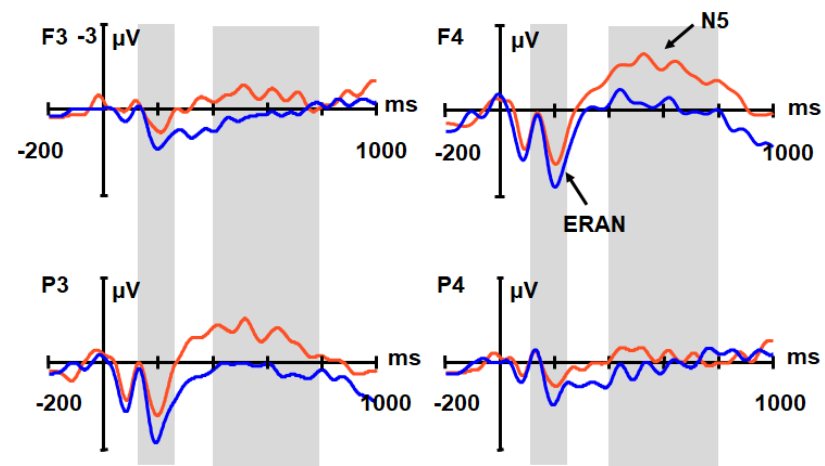

Irregular

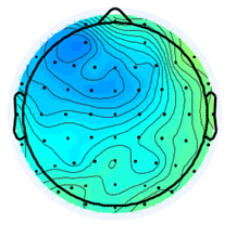

$110-250 \mathrm{~ms}$

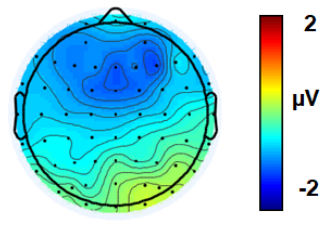

400-800 ms 
Table 1. Participants' characteristics and mean scores of the MBEA and pitch thresholds for each group in Experiment 1. Standard deviation values are shown in parentheses.

\begin{tabular}{llll}
\hline & Amusic $(\mathrm{n}=16)$ & Control $(\mathrm{n}=16)$ & t-test \\
\hline Mean age $(S D)$ & $24.06(2.17)$ & $23.44(2.22)$ & $p=.437$ \\
Sex & $10 \mathrm{~F}, 6 \mathrm{M}$ & $11 \mathrm{~F}, 5 \mathrm{M}$ & \\
Years education $(S D)$ & $16.94(1.95)$ & $17.06(2.26)$ & $p=.868$ \\
Melodic score of MBEA $(S D)$ & $18.42(2.03)$ & $27.48(1.05)$ & $p<.001$ \\
Global score of MBEA $(S D)$ & $18.75(1.40)$ & $27.32(0.77)$ & $p<.001$ \\
d' of MBEA $(S D)$ & $0.85(0.31)$ & $2.77(0.26)$ & $p<.001$ \\
Pitch detection threshold & $1.62(1.61)$ & $0.44(0.31)$ & $p=.008$ \\
Pitch direction discrimination threshold & $4.47(1.67)$ & $2.33(2.32)$ & $p=.003$ \\
\hline
\end{tabular}

Note: $\mathrm{F}=$ female; $\mathrm{M}=$ male. The "Melodic score of MBEA" refers to the mean score of the three pitch-based subtests. 
Table 2. Participants' characteristics and mean scores of the MBEA and pitch thresholds for each group in Experiment 2. Standard deviation values are shown in parentheses.

\begin{tabular}{llll}
\hline & Amusic $(\mathrm{n}=16)$ & Control $(\mathrm{n}=16)$ & $\mathrm{t}$-test \\
\hline Mean age $(S D)$ & $24.00(2.10)$ & $23.56(1.79)$ & $p=.530$ \\
Sex & $10 \mathrm{~F}, 6 \mathrm{M}$ & $10 \mathrm{~F}, 6 \mathrm{M}$ & \\
Years education $(S D)$ & $17.00(2.00)$ & $17.06(1.88)$ & $p=.928$ \\
Melodic score of MBEA $(S D)$ & $18.35(1.97)$ & $27.60(0.94)$ & $p<.001$ \\
Global score of MBEA $(S D)$ & $18.58(1.36)$ & $27.38(0.92)$ & $p<.001$ \\
d' of MBEA $(S D)$ & $0.82(0.32)$ & $2.79(0.30)$ & $p<.001$ \\
Pitch detection threshold & $1.50(1.63)$ & $0.42(0.31)$ & $p=.015$ \\
Pitch direction discrimination threshold & $4.46(1.70)$ & $2.01(2.31)$ & $p=.002$ \\
\hline
\end{tabular}

Note: $\mathrm{F}=$ female; $\mathrm{M}=$ male. The "Melodic score of MBEA" refers to the mean score of the three pitch-based subtests. 